OPEN

SUBJECT AREAS:

RNAI

GENE EXPRESSION

Received

23 January 2015

Accepted

9 March 2015

Published

15 April 2015

Correspondence and requests for materials should be addressed to

E.A.M. (eric.miska@

gurdon.cam.ac.uk)

* Current address: MRC Clinical Sciences

Centre, Imperial College London, London, United Kingdom.

\section{E. coli OxyS non-coding RNA does not trigger RNA $i$ in $C$. elegans}

\author{
Alper Akay ${ }^{1,2}$, Peter Sarkies ${ }^{1,2 *}$ \& Eric A. Miska ${ }^{1,2}$
}

'Wellcome Trust Cancer Research UK Gurdon Institute, University of Cambridge, Tennis Court Rd, Cambridge CB2 1QN, UK,
2Department of Biochemistry and Department of Genetics, University of Cambridge, Tennis Court Rd, Cambridge CB2 1QN, UK.

The discovery of RNA interference (RNAi) in C. elegans has had a major impact on scientific research, led to the rapid development of RNAi tools and has inspired RNA-based therapeutics. Astonishingly, nematodes, planaria and many insects take up double-stranded RNA (dsRNA) from their environment to elicit RNAi; the biological function of this mechanism is unclear. Recently, the E. coli OxyS non-coding RNA was shown to regulate gene expression in $C$. elegans when $E$. coli is offered as food. This was surprising given that $C$. elegans is unlikely to encounter $E$. coli in nature. To directly test the hypothesis that the E. coli OxyS non-coding RNA triggers the $C$. elegans RNAi pathway, we sequenced small RNAs from $C$. elegans after feeding with bacteria. We clearly demonstrate that the OxyS non-coding RNA does not trigger an RNAi response in $C$. elegans. We conclude that the biology of environmental RNAi remains to be discovered.

T he biology underlying RNAi is still only partially understood. Bacteria engineered to express dsRNA homologous to C. elegans genes can induce RNAi when fed to the animals ${ }^{1}$; transgenic plants engineered to express dsRNA against genes of plant-parasitic nematodes kill these nematodes when used as hosts ${ }^{2}$. Why any animal, or indeed C. elegans in particular, should respond to environmental dsRNA, is an exciting question that remains unanswered. We recently discussed some potential roles of environmental RNAi in animal to animal communication ("social RNA").

Environmental RNAi in C. elegans is a multi-step process (Fig. 1a). First, dsRNA molecules from food are taken up by intestinal cells, a process that requires the dsRNA transporter SID- $2^{4}$. dsRNA and/or RNAi intermediates can move between cells and tissues of the animal, a phenomenon referred to as systemic RNAi, which requires SID-1 RNA channel and other protein factors ${ }^{5,6}$. Within cells dsRNA is processed by the DICER endonuclease (DCR-1)/RDE-4 complex, which generates short dsRNA ${ }^{7-9}$. Either of the strands of the short dsRNA, the $1^{\circ}$ siRNA, is incorporated into the Argonaute protein RDE- $1^{10,11}$ and finds its target through primary sequence complementarity (Watson-Crick base-pairing). Upon target recognition, the RDE- $1 / 1^{\circ}$ siRNA complex recruits RNA-dependent RNA polymerases (RdRPs) to generate $2^{\circ}$ siRNAs, also known as $22 \mathrm{G}-\mathrm{RNAs}^{12}$. $22 \mathrm{G}-\mathrm{RNAs}$ associate with a different class of Argonaute proteins (WAGOs) and form the active RNAi effector complex. 22GRNAs lead to gene silencing at both the post-transcriptional and transcriptional level ${ }^{13,14}$. Due to the RdRP amplification step, 22G-RNAs are highly abundant and are a key signature of RNAi in C. elegans (Fig. 1b).

C. elegans is a common animal model and the standard food source in the laboratory is $E$. coli. In nature $C$. elegans is associated with soil and decaying organic matter and is likely a generalist feeding on microorganisms such as bacteria ${ }^{15}$. Curiously, the E. coli stress-induced non-coding RNA OxyS ${ }^{16}$ has a 17 nucleotides (nt) region complementary to the sequence of the che-2 gene of $C$. elegans ${ }^{17}$ (Fig. 2). Recently, Liu et al. ${ }^{17}$ reported that the OxyS non-coding RNA causes down-regulation of the che-2 mRNA in C. elegans when expressed in the E. coli food. According to the genetic analysis of Liu et al. ${ }^{17}$, OxyS-dependent down-regulation of che-2 requires some of the components of the RNAi and miRNA pathways in C. elegans. Specifically, $d c r-1$ and $r d e-4$, essential for long dsRNA processing during RNAi ${ }^{8,9}, r d e-1$, the $1^{\circ}$ siRNA binding Argonaute ${ }^{11,18}$, and $\operatorname{alg}-1$, a microRNA (miRNA) pathway gene ${ }^{10}$, were all reported to be required for $c h e-2$ mRNA regulation ${ }^{17}$. Surprisingly, the systemic RNAi genes sid- $1^{5}$ and sid- $2^{4}$ were found to be dispensible ${ }^{17}$. The authors concluded that the OxyS RNA elicits an RNAi response in C. elegans that results in gene expression changes and behavioral responses.

\section{Results}

To test this hypothesis directly, we fed three different E. coli strains to C. elegans either for one or three generations: an OxyS over-expressing strain (AZ121), an OxyS deletion strain (GS035) and the common C. elegans food strain (HB101). We isolated total RNA from young adult stage animals and prepared 5' independent small RNA 


\section{C. elegans Exo-RNAi Pathway}

a

b

Extra cellular long dsRNA

5' monophosphate

$5^{\prime}$ triphosphate

SID-2 uptake from environment

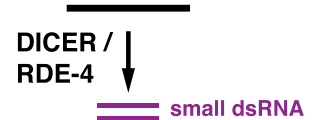

primary siRNAs (5' monophosphate) $\downarrow$ RNA dependent RNA polymerase

secondary siRNAs (5' triphosphate)
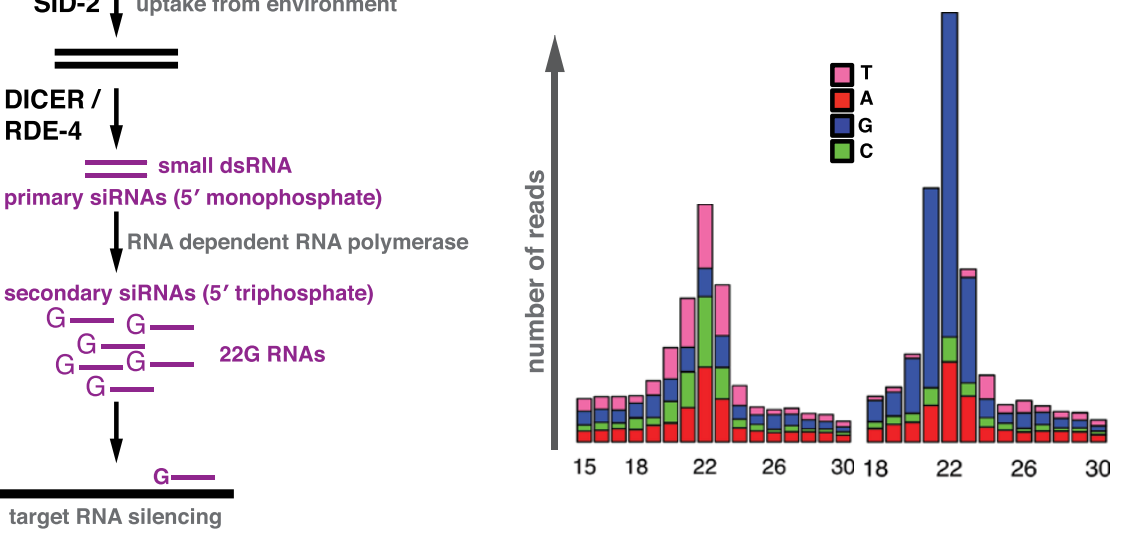

Figure $1 \mid$ C. elegans exogenous RNAi pathway. (a) Extracellular long dsRNA is taken up by the intestinal cells through SID-2 RNA transporter. Intracellular long dsRNAs are processed into short dsRNAs by the DICER/RDE-4 complex. Either of the strands of the short dsRNA can become the primary siRNA. Primary siRNAs form a complex with the Argonaute protein RDE-1 and find target sequences by sequence complementarity.

Recruitment of RNA dependent RNA polymerase generates secondary siRNAs (22G-RNAs). (b) Different cloning strategies can differentiate between the primary and secondary siRNAs depending on their $5^{\prime}$ phosphate state.

libraries which enables the sequencing of both $1^{\circ}$ and $2^{\circ} \operatorname{siRNAs}{ }^{12}$ (Fig. 1b). We then mapped the small RNA reads against the OxyS non-coding RNA. We did not detect any small RNAs mapping to the OxyS RNA in animals fed with either the OxyS deletion strain or the HB101 E. coli strain (data not shown). In animals fed with the AZ121 strain, small RNAs mapping to the OxyS RNA show a peak at 21$22 \mathrm{nt}$ both in one or three generation fed animals (Fig. 3, top two panels). However, these small RNAs map exclusively to the $3^{\prime}$ region of the OxyS RNA. We did not detect any small RNAs mapping to the region of the OxyS RNA that is predicted to share sequence similarity to the che-2 mRNA (Fig. 3, bottom two panels). To test whether Dicer activity was involved in the generation of these small RNAs, we repeated the feeding experiment using $r d e-4$ mutant animals (Fig. 3, top and bottom third panels). Small RNA reads from $r d e-4$ mutants showed a very similar pattern to those from wild-type animals with abundant 21-22 nt RNAs mapping exclusively to the 3' region of the OxyS RNA. These results indicate that the DCR-1/ RDE-4 complex is not required for the generation of these 2122 nt small RNAs which originate from the OxyS RNA. Next, we checked for 22G-RNAs, which are the effectors of C. elegans RNAi pathway. We did not observe any 22G-RNAs mapping either against the OxyS RNA or the che-2 mRNA in our 5' independent small RNA libraries, thus suggesting that there is no small RNA mediated silencing of che-2. Consistently, we also did not observe any down-regulation of the che-2 mRNA upon AZ121 feeding (Fig. 4).

Given the lack of engagement of the RNAi pathway, we speculated that the small RNAs mapping to the OxyS RNA might be generated by the bacteria and not by $C$. elegans. To test this we sequenced the small RNAs from the E.coli strains alone. In the AZ121 strain, we detected a very similar pattern of 21-22 nt small RNAs, mostly mapping to the $3^{\prime}$ end of the OxyS RNA and more abundant than the reads detected from the sequenced animals (Fig. 3, rightmost panels). Thus small RNAs arising from the OxyS RNA are made within the bacteria and are not generated by the $C$. elegans RNAi pathway. We believe that these small RNAs are likely degradation products of the OxyS RNA and do not enter the RNAi pathway. We also mapped all the small RNAs to the E. coli genome to make sure that the OxyS RNA is expressed (Fig. 5). In AZ121 strain, small RNA reads mapping to the OxyS RNA can be detected in abundance (Fig. 5, top panel red line). In contrast, GS035 strain has no reads mapping to the OxyS RNA.

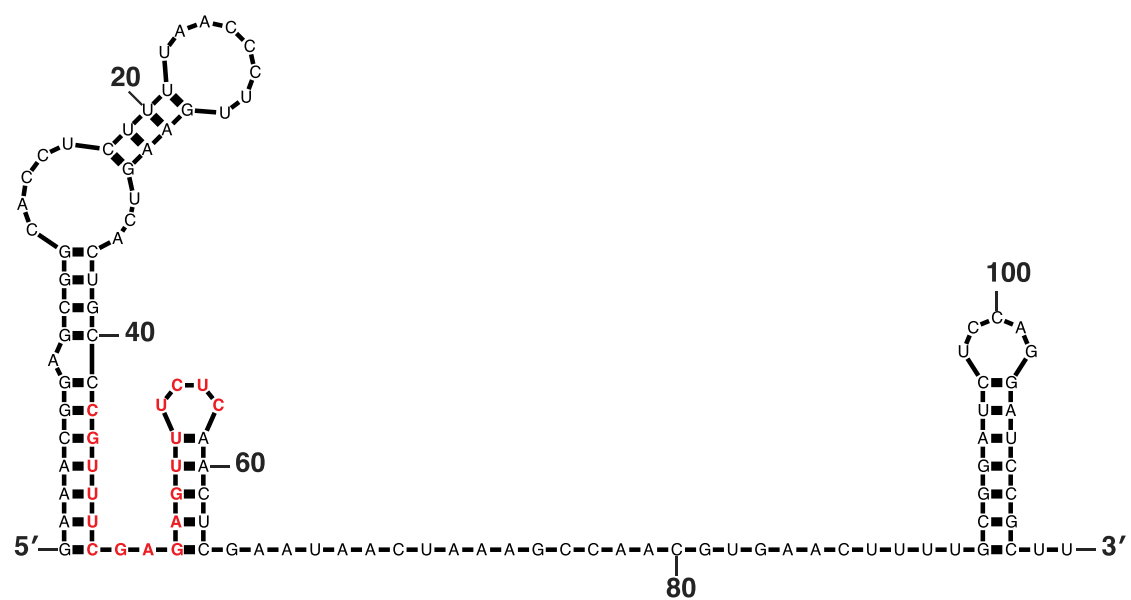

Figure $2 \mid$ E. coli OxyS non-coding RNA. Nucleotides complementary to the C. elegans che-2 gene are highlighted in red. 

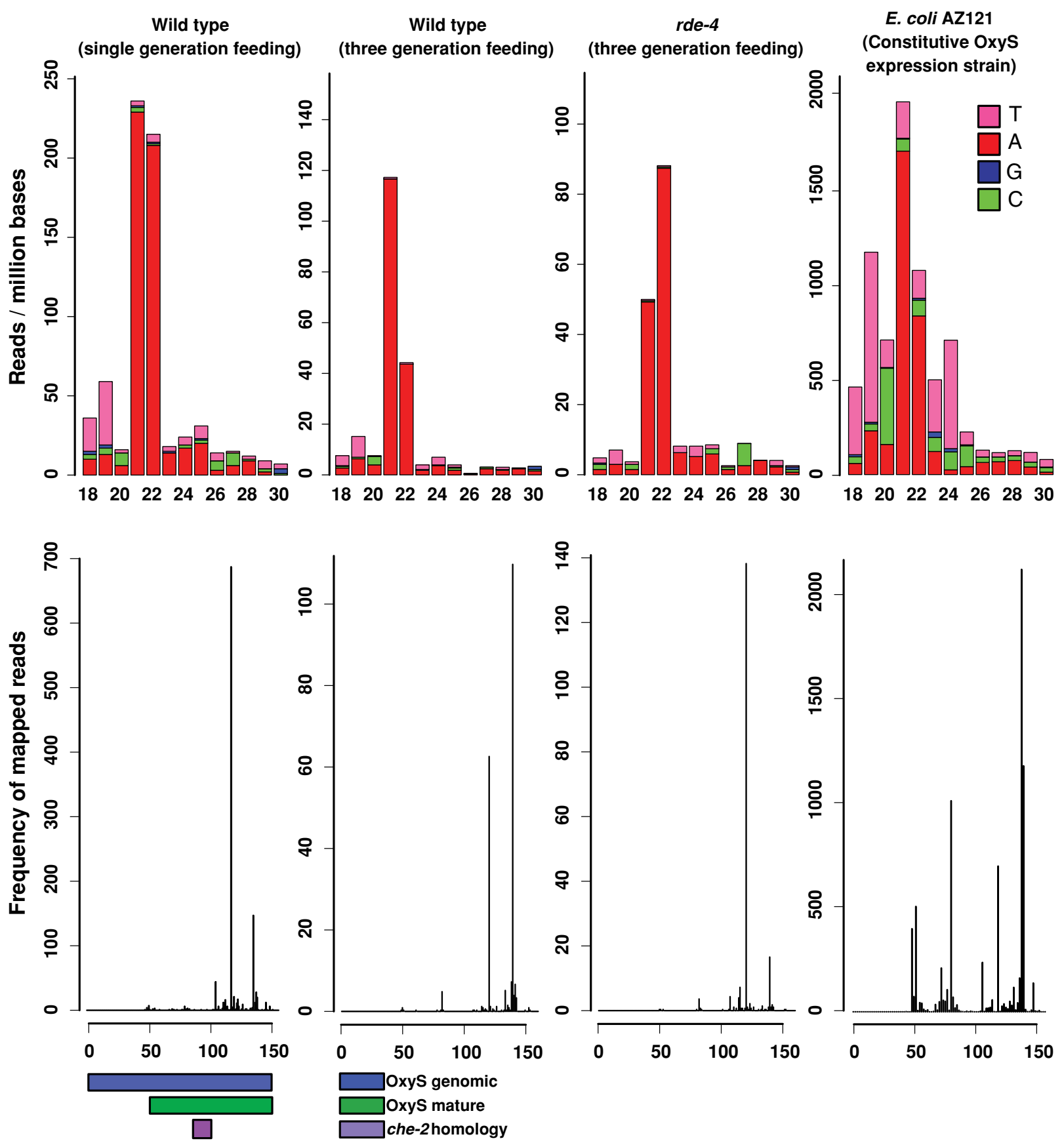

Figure $3 \mid$ E. coli strain AZ121 with constitutive OxyS non-coding RNA expression does not induce RNAi in C. elegans upon feeding. Small RNAs from one or three generation fed C. elegans larvae and the small RNAs from E. coli AZ121 (constitutive OxyS expression strain) are mapped onto the OxyS RNA. Upper panels show the size and nucleotide distribution of mapped reads. Lower panels show the frequency of mapped reads along the OxyS non-coding RNA. Colored bars indicate the genomic, mature and che-2 complementary sequences of the OxyS RNA.

\section{Discussion}

Here we show that small RNA sequencing can efficiently be used to elucidate possible regulatory roles of non-coding RNAs in C. elegans. Our analysis of the OxyS RNA as a trigger for RNAi in C. elegans clearly shows that OxyS non-coding RNA does not enter the canonical RNAi pathway in C. elegans upon feeding. We suggest that the previously described phenotypes in C. elegans upon OxyS non-coding RNA feeding ${ }^{17}$ are more likely to be caused by the differences within the bacterial strains. It is known that different bacteria can affect the physiology of the nematodes with respect to foraging, metabolism and ageing ${ }^{19,20}$. It remains possible that RNA produced by different bacteria in nature enters the C. elegans RNAi pathway; however, our results suggest that sequencing of the small RNA popu- lations in C. elegans is essential to confirm this. We conclude that the biology of environmental RNAi remains to be discovered.

\section{Methods}

E. coli HB101, AZ121 and GS035 strains were grown in LB media containing no antibiotics, ampicillin $(100 \mu \mathrm{g} / \mathrm{ml})$ or chloramphenicol $(25 \mu \mathrm{g} / \mathrm{ml})$ respectively $(\mathrm{OD} 595=0.6-0.8)$. Cultures were centrifuged and the re-suspended pellets were seeded onto NGM plates. For one generation feeding experiments, animals were placed on the seeded NGM plates and allowed to grow until young adult stage. For three generation feeding experiments, L1 larval stage animals were placed on the seeded NGM plates and allowed to grow for three generations. Total RNA from harvested animals was isolated using the TriSure reagent (Bioline). Small RNA sequencing libraries of $C$. elegans were prepared using the NEBNext Multiplex Small RNA Library Prep set (NEB) and the small RNA sequencing libraries of E. coli are prepared using the TruSeq Small RNA set (Illumina). Libraries were either sequenced 


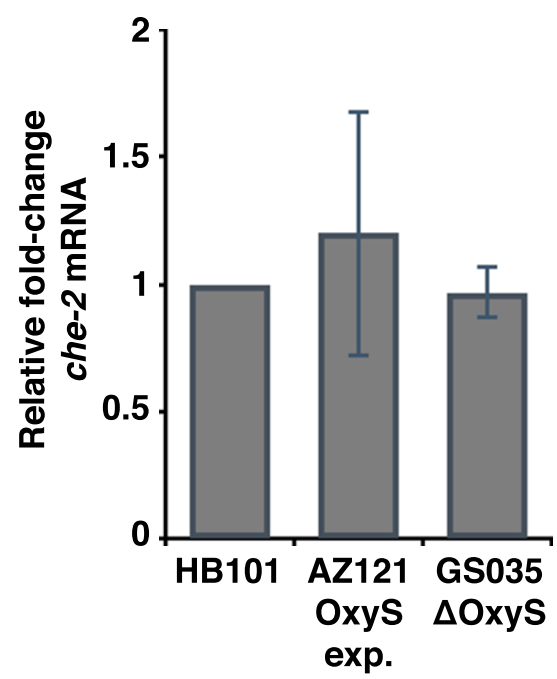

Figure $4 \mid$ che- 2 mRNA is not down-regulated upon OxyS RNA feeding. Relative fold-change of che-2 mRNA in animals fed with either the constitutive OxyS expression strain (E. coli AZ121) or the $\Delta$ OxyS (E. coli GS035). Fold-change is calculated relative to the che-2 mRNA levels in animals fed with the wild type E. coli strain HB101.
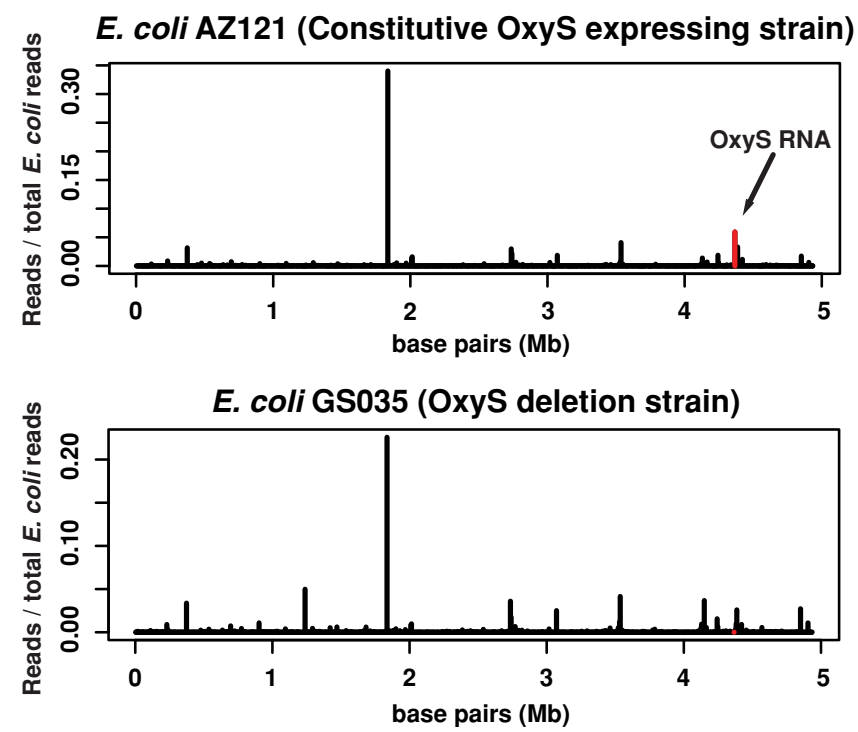

Figure $5 \mid$ small RNA reads mapping to the $E$. coli genome. Small RNA sequencing reads from animals fed with either E. coli AZ121 (upper panel) or E. coli GS035 (lower panel) are mapped to the E. coligenome in windows of 5,000 bases. Red bar in the upper panel highlights the reads mapping to the OxyS transcript. There were no reads mapping to the OxyS transcript in the $\Delta$ OxyS GS035 strain (lower panel).

on a MiSeq or a HiSeq 1500 machine (Illumina). Data analysis was performed as previously described ${ }^{21}$. Small RNA-seq data is available through NCBI-GEO with accession number GSE64990. qRT-PCR is performed with primers described in Liu et $\mathrm{al}^{17}$. OxyS RNA structure is determined using the Mfold web server ${ }^{22}$.

1. Timmons, L., Court, D. L. \& Fire, A. Ingestion of bacterially expressed dsRNAs can produce specific and potent genetic interference in Caenorhabditis elegans. Gene 263, 103-112 (2001).
2. Yadav, B. C., Veluthambi, K. \& Subramaniam, K. Host-generated double stranded RNA induces RNAi in plant-parasitic nematodes and protects the host from infection. Mol. Biochem. Parasitol. 148, 219-222 (2006).

3. Sarkies, P. \& Miska, E. A. Molecular biology. Is there social RNA? Sci. (New York, NY) 341, 467-468 (2013).

4. Winston, W. M., Sutherlin, M., Wright, A. J., Feinberg, E. H. \& Hunter, C. P. Caenorhabditis elegans SID-2 is required for environmental RNA interference. Proc. Natl. Acad. Sci. U. S. A. 104, 10565-10570 (2007).

5. Feinberg, E. H. \& Hunter, C. P. Transport of dsRNA into cells by the transmembrane protein SID-1. Sci. (New York, NY) 301, 1545-1547 (2003).

6. Hinas, A., Wright, A. J. \& Hunter, C. P. SID-5 is an endosome-associated protein required for efficient systemic RNAi in C. elegans. Curr. Biol. 22, 1938-1943 (2012).

7. Knight, S. W. \& Bass, B. L. A role for the RNase III enzyme DCR-1 in RNA interference and germ line development in Caenorhabditis elegans. Sci. (New York, NY) 293, 2269-2271 (2001).

8. Parker, G. S., Eckert, D. M. \& Bass, B. L. RDE-4 preferentially binds long dsRNA and its dimerization is necessary for cleavage of dsRNA to siRNA. RNA (New York, NY) 12, 807-818 (2006).

9. Tabara, H., Yigit, E., Siomi, H. \& Mello, C. C. The dsRNA binding protein RDE-4 interacts with RDE-1, DCR-1, and a DExH-box helicase to direct RNAi in C. elegans. Cell 109, 861-871 (2002).

10. Grishok, A. et al. Genes and mechanisms related to RNA interference regulate expression of the small temporal RNAs that control C. elegans developmental timing. Cell 106, 23-34 (2001).

11. Yigit, E. et al. Analysis of the C. elegans Argonaute family reveals that distinct Argonautes act sequentially during RNAi. Cell 127, 747-757 (2006).

12. Pak, J. \& Fire, A. Distinct populations of primary and secondary effectors during RNAi in C. elegans. Sci. (New York, NY) 315, 241-244 (2007).

13. Guang, S. et al. Small regulatory RNAs inhibit RNA polymerase II during the elongation phase of transcription. Nature 465, 1097-1101 (2010).

14. Guang, S. et al. An Argonaute Transports siRNAs from the Cytoplasm to the Nucleus. Sci. (New York, NY) 321, 537-541 (2008).

15. Félix, M.-A. \& Braendle, C. The natural history of Caenorhabditis elegans. Curr. Biol. 20, R965-9 (2010).

16. Altuvia, S., Weinstein-Fischer, D., Zhang, A., Postow, L. \& Storz, G. A small, stable RNA induced by oxidative stress: role as a pleiotropic regulator and antimutator. Cell 90, 43-53 (1997).

17. Liu, H. et al. Escherichia coli noncoding RNAs can affect gene expression and physiology of Caenorhabditis elegans. Nat. Commun. 3, 1073 (2012).

18. Tabara, H. et al. The rde-1 gene, RNA interference, and transposon silencing in C. elegans. Cell 99, 123-132 (1999).

19. Brooks, K. K., Liang, B. \& Watts, J. L. The influence of bacterial diet on fat storage in C. elegans. PLoS One 4, e7545 (2009).

20. Melo, J. A. \& Ruvkun, G. Inactivation of conserved C. elegans genes engages pathogen- and xenobiotic-associated defenses. Cell 149, 452-466 (2012).

21. Ashe, A. et al. piRNAs Can Trigger a Multigenerational Epigenetic Memory in the Germline of C. elegans. Cell 150, 88-99 (2012).

22. Zuker, M. Mfold web server for nucleic acid folding and hybridization prediction. Nucleic Acids Res. 31, 3406-3415 (2003).

\section{Acknowledgments}

We thank Gisela Storz for sharing E. coli AZ121 and GS035 strains and Sylviane Moss for running the sequencing machines. PS is funded by a research fellowship from the Gonville and Caius College, University of Cambridge. AA and EAM are supported by a Cancer Research UK programme grant to EAM.

\section{Author contributions}

A.A. initiated the project and performed the experiments. P.S. contributed to the experiments and analyzed the data. A.A., P.S. and E.A.M. developed the concepts and wrote the manuscript.

\section{Additional information}

Competing financial interests: The authors declare no competing financial interests. How to cite this article: Akay, A., Sarkies, P. \& Miska, E.A. E. coli OxyS non-coding RNA does not trigger RNAi in C. elegans. Sci. Rep. 5, 9597; DOI:10.1038/srep09597 (2015).

This work is licensed under a Creative Commons Attribution 4.0 International License. The images or other third party material in this article are included in the article's Creative Commons license, unless indicated otherwise in the credit line; if the material is not included under the Creative Commons license, users will need to obtain permission from the license holder in order to reproduce the material. To view a copy of this license, visit http://creativecommons.org/licenses/by/4.0/ 